\title{
Relationship between Level of Physical Activity with Self-Esteem, Body Image Concern and Body Appreciation in Veterans
}

\section{ART I C L E INF O}

\section{Article Type}

Descriptive Study

\section{Authors}

Heydari $\mathrm{Y}^{1} M S C$

Samadi H.*2 PhD,

Dehghan Manshadi M. ${ }^{3} \mathrm{PhD}$

\section{A B S T R A C T}

Aims Paying attention to mental and physical health is very important for all society sections, especially veterans. This study aimed to investigate the relationship between physical activity and self-esteem, body image concern, and veterans' self-esteem.

Instruments \& Methods In this descriptive study, the statistical sample consisted of 100 male veterans who did not have any acute problems in physical and physical conditions and were selected by the available sampling method. The instruments used in this study included a demographic questionnaire, shark physical activity, body image concern, self-esteem, and physical self-worth. Data analysis was performed using the Pearson correlation coefficient and multiple regression analysis with simultaneous input of variables and SPSS 23 software. Findings Physical activity had a positive relationship with self-esteem $(r=0.442 ; p<0.01)$ and physical self-worth $(\mathrm{r}=0.532 ; \mathrm{p}<0.01)$, and had a negative relationship with body image concern $(\mathrm{r}=-0.373 ; \mathrm{p}<0.01)$. P). Only the physical self-worth variable $(\mathrm{t}=2.726 ; \mathrm{p}<0.01)$ predicted the physical activity.

Conclusion There is a relationship between physical activity with self-esteem, physical selfworth, and body image concern. the variable of physical self-worth has the ability to predict the variable of physical activity.

Keywords Physical Activity; Self-Esteem; Concern for Body Image; Body Appreciation; Veterans
${ }^{1}$ Educational Science Department, Psychology and Educational Sciences Faculty, Yazd University, Yazd, Iran

${ }^{2}$ Physical Education and Sports Science Department, Psychology and Educational Sciences Faculty, Yazd University, Yazd, Iran

${ }^{3}$ Educational Science Department, Farhangian University, Tehran, Iran

\section{*Correspondence}

Address: Psychology and Educational Sciences Faculty, Yazd University, University Blvd., Safaieh, Yazd, Iran. Phone: +98 (21) 71732256

Fax: +98(35) 31232222 samadih@yazd.ac.ir

\section{Article History}

Received: January 21, 2020

Accepted: May 14, 2020

ePublished: June 17, 2020

\section{I T A T I O N L I N K S}

[1] Comparison of body dimensions satisfaction ... [2] The effect of internal attentional focus ... [3] Comparison of body image anxiety of athletic ... [4] Investigating adolescent stress and ... [5] The association between body esteem ... [6] Investigating mental status and body ... [7] Fear of negative evaluation and the ... [8] The relationship between women's ... [9] Determinants of body-image ... [10] Predictive factors of self-esteem ... [11] Investigate the relationship between ... [12] Standardization of self-worthiness questionnaires ... [13] The relationship between self-worth and ... [14] Evaluation of depression and its relationship ... [15] Relationship between physical activity and job ... [16] Physical activity in the prevention and amelioration ... [17] Physical activity, fitness, and physical selfconcept ... [18] Self-belief does make a difference: A reciprocal ... [19] Effect of selected mental practice on ... [20] Comparing self-esteem and self-concept ... [21] The comparison and relationship between ... [22] Development of the body image concern ... [23] The relationship between body ... [24] Global self-esteem and specific self-esteem ... [25] The Comparison of coping styles of stress ... [26] Reliability and factor validity of the 7-item ... [27] The Comparison of brain cognition function ... [28] Persian psychometric properties of homework ... [29] Relationship between spiritual health with ... [30] Self-Presentation in Exercise Contexts ... [31] Study of the relationship between regular physical ... [32] Study of relationship between physical ... [33] Effect of strength exercise on physical ... [34] Body image and self-esteem among ... [35] Relationship of physical self-concept ... [36] The effects of physical fitness training ... [37] The effect of gender and level of physical ... [38] Construct validity of physical self-description ... 
مى كذارد[3]. نظر به اينكه سلامت جسمانى و روانى هر كدام به سهم

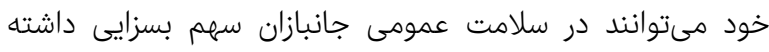

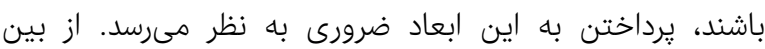

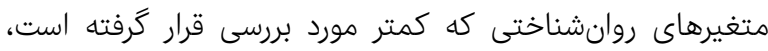
اصطلاح "تصوير بدن" (Body Image) است. تصوير بدن يك سازه

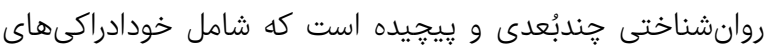

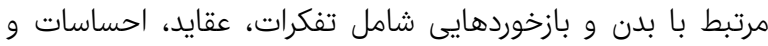

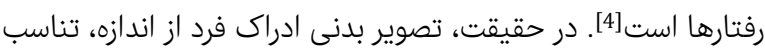

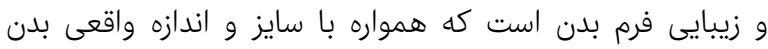

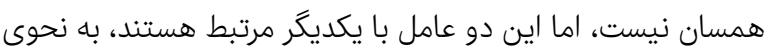

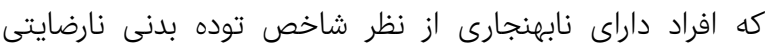

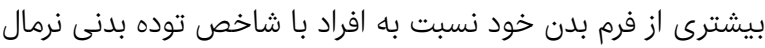

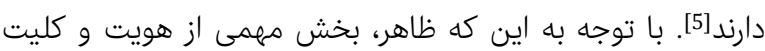

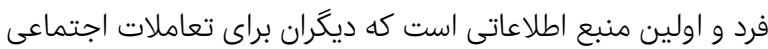

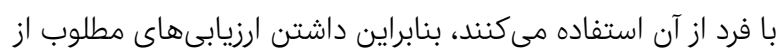

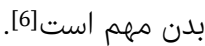

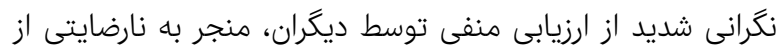

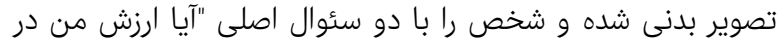

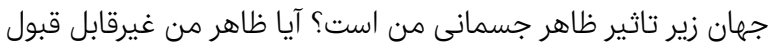

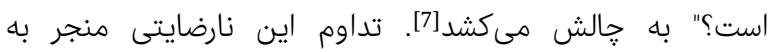

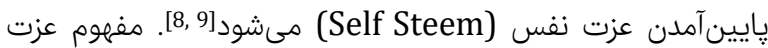
نفس از مفاهيم مهم و كليدى در نظريات و مطالعات خون خود محسوب

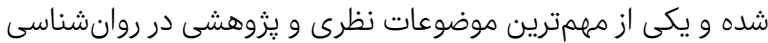

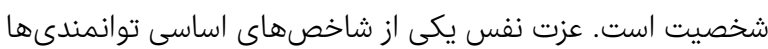

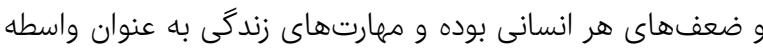

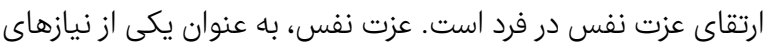

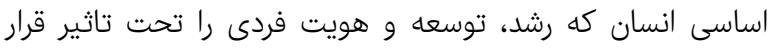

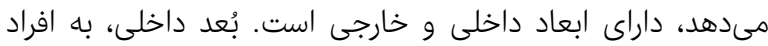

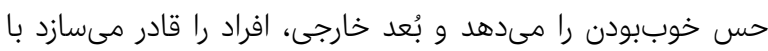

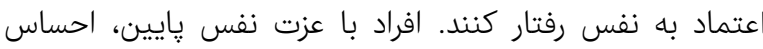

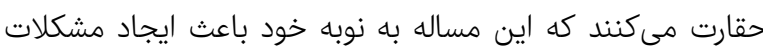

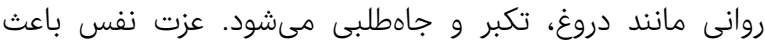

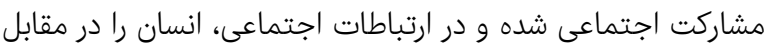
اضطراب و اختلالات جسمانى حمايت نموده و شيوههاى مقان إنابله

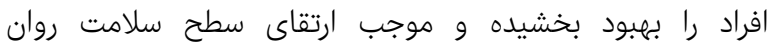

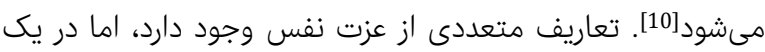

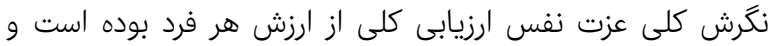

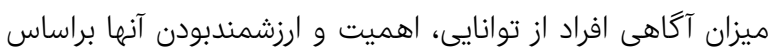

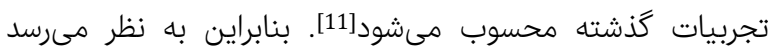

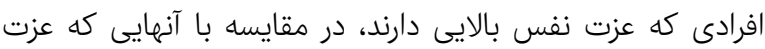

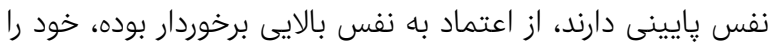

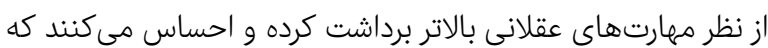

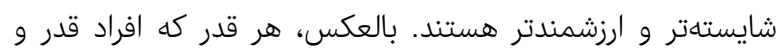
منزلت و ارزش خود را بدانند، به همان نسبت اعتماد به خويشتن
ارتباط بين ميزان فعاليت بدنى با عزت نفس، نكرانى از تصوير بدن و خود ارزشمندى جسمانى جانبازان

MSc ياسر حيدرى

كروه علوم تربيتى، دانشكده روانشناسى و علوم تربيتى، دانشكاه يزد، يزد، ايران

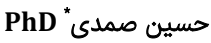

كروه تربيت بدنى و علوم ورزشى، دانشكده روانشناسى و علوم تربيتى، دانشكاه

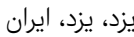

Pنصور دهقان منشادى Pيزي كروه علوم تربيتى، دانشگًاه فرهنگيان، تهران، ايران

جكيده اهداف: توجه به سلامت روانى و جسمانى براى تمام اقشار جامعه به خصوص

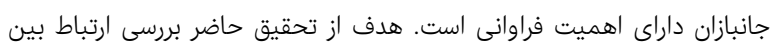

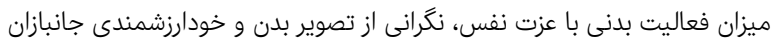

ابزار و روشها: يزوهش حاضر از نوع توصيفى است. نمونه آمارى يزوهش حاضر

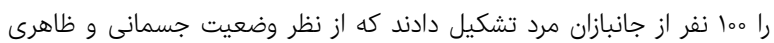

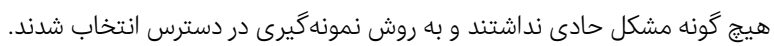

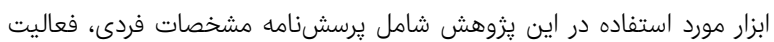

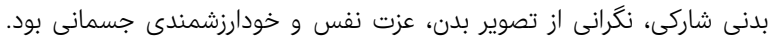

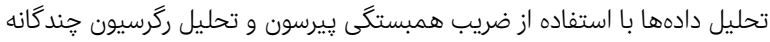

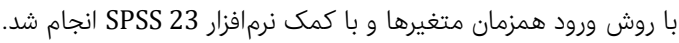

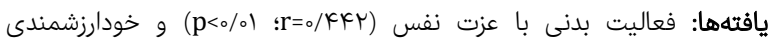

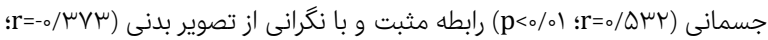

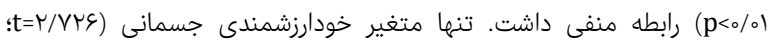

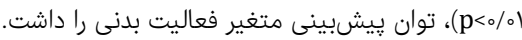

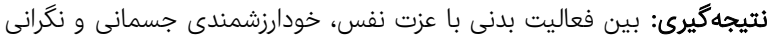

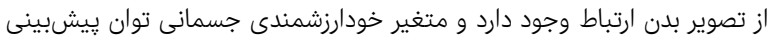

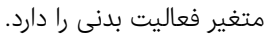

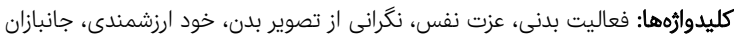

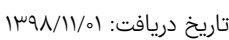

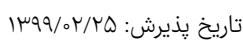

'نويسنده مسئول: samadih@yazd.ac.ir

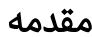

توجه به وضعيت سلامت جسمانى و روانشناختى، اجتماعى و

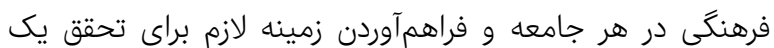

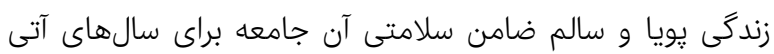

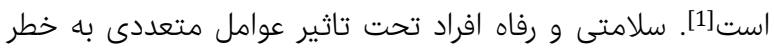

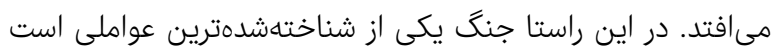

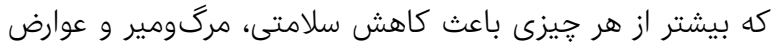

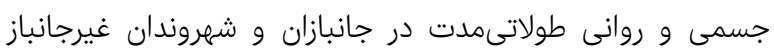

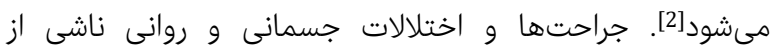

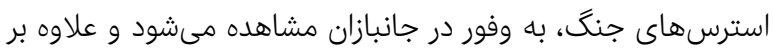

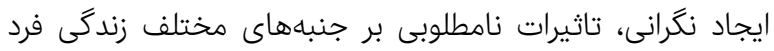


ارتباط بين ميزان فعاليت بدنى با عزت نفس، نكرانى از تصوير بدن و خود ارزشمندى جسمانى جانبازان هـا إسا

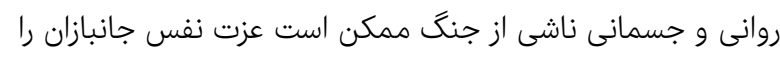

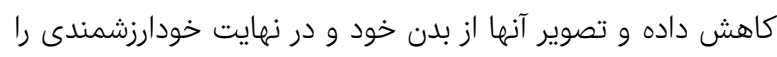

$$
\text { به طور منفى تغيير دهد. }
$$

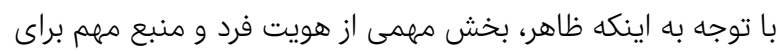

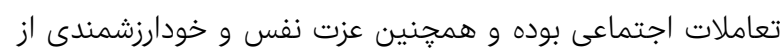

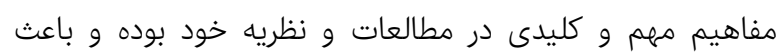

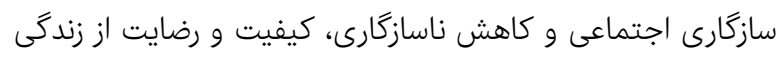

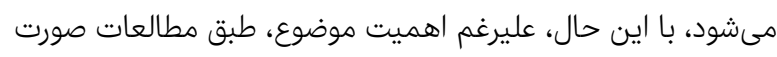

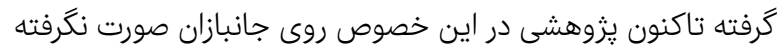

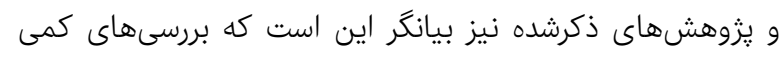

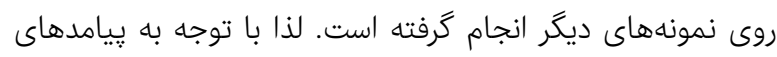

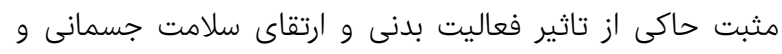

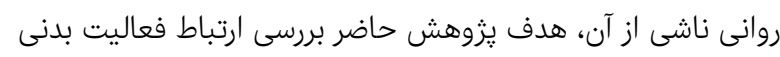

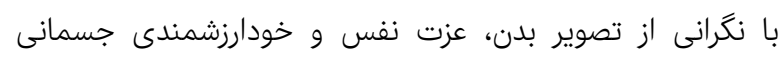

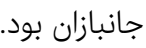

\section{ابزار و روشها}

يثزوهش حاضر از نوع توصيفى-ميدانى است و در سال هوسا روى رونا

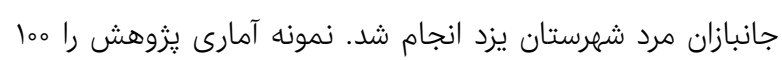

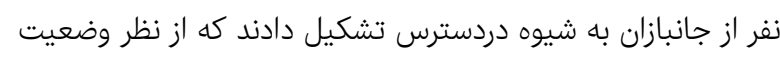

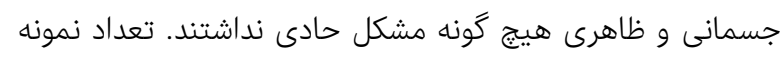

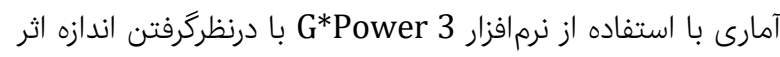

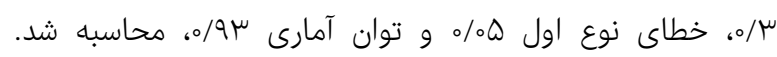

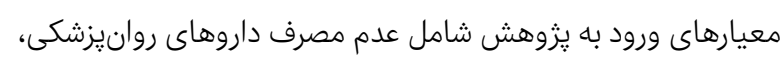

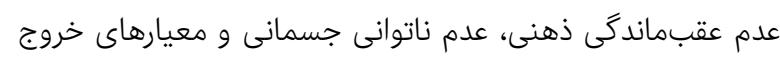

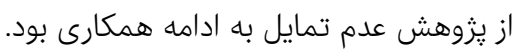

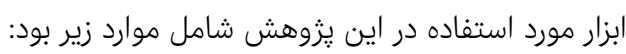

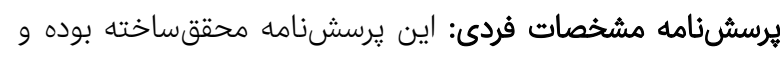

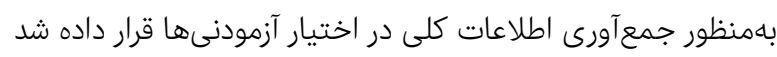

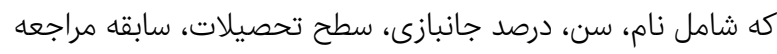

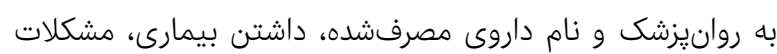
جسمانى و غيره بود.

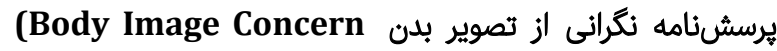
ته :Inventory; BICI)

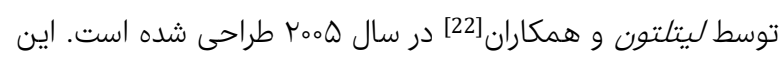
يرسشنامه نارضايتى از تصوير بدنى را در دو بُعد نارضايتى از ظاهر

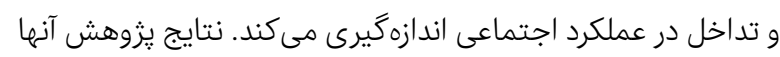

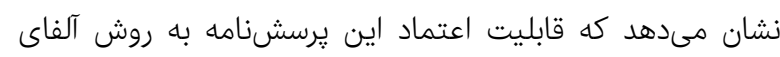

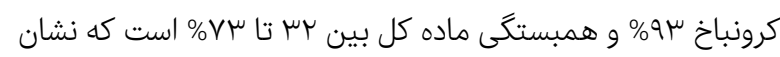

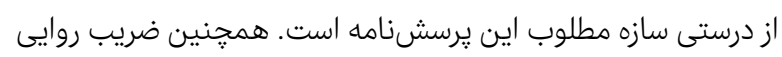

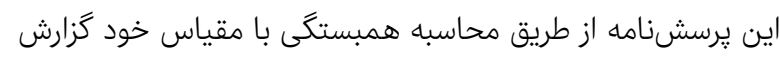

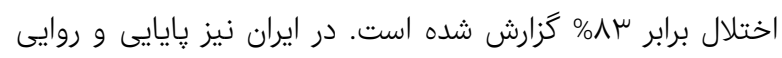

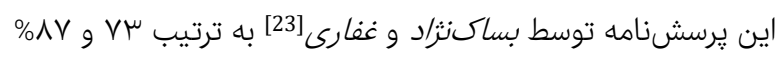
كزارش شده است.
آنها افزوده شده و در نهايت از عزت نفس بالاترى برخوردار خواهند

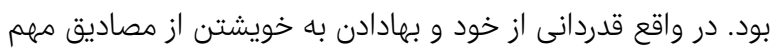

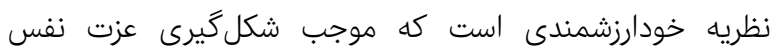

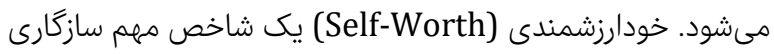

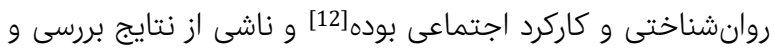

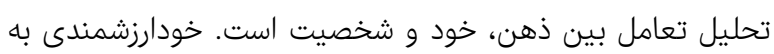

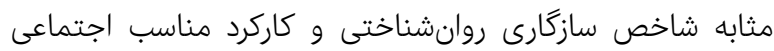

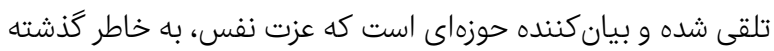

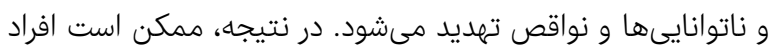

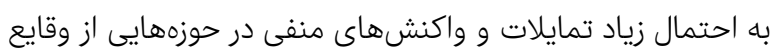

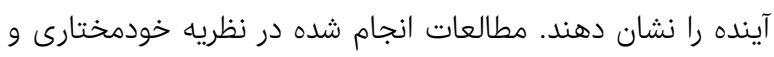

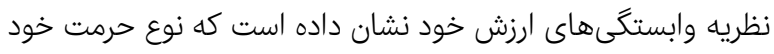

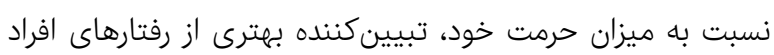

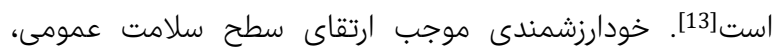

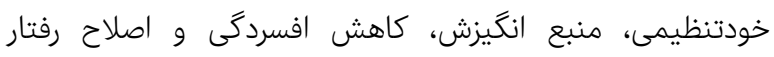

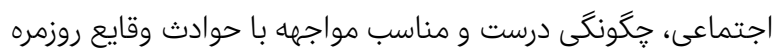
زندگى بوده كه در سطوح مختلف زندگى فردى، خانوادگى، آموزشى دوسى

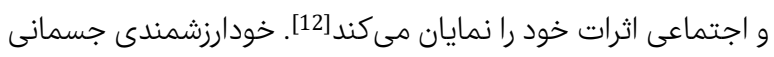

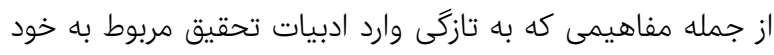

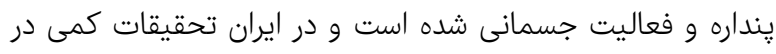

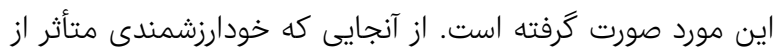

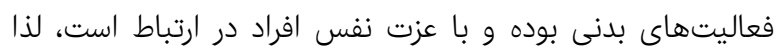

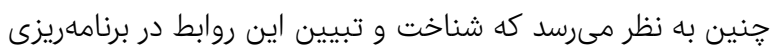
و هدفگذارى مسئولين و متخصصين علوم رفتارى اهميت بسزايى

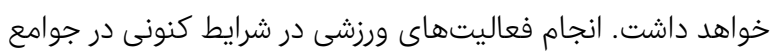

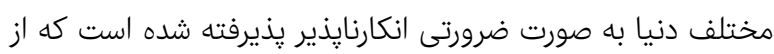

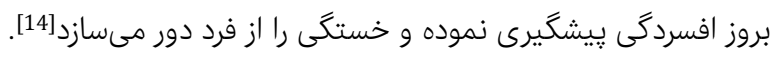

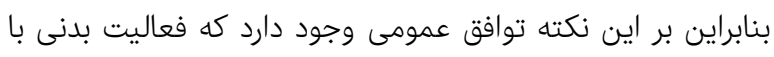

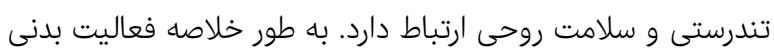

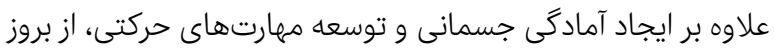

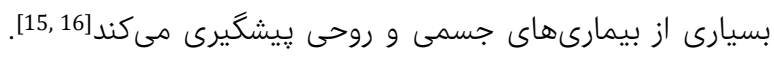

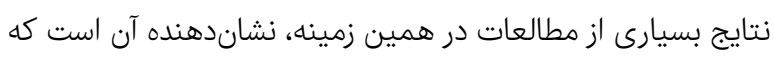

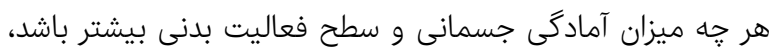

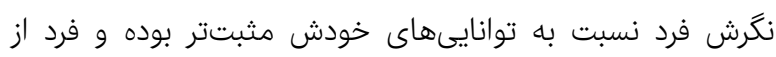

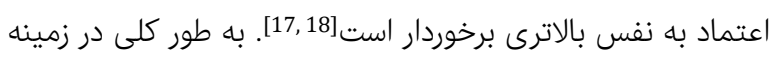

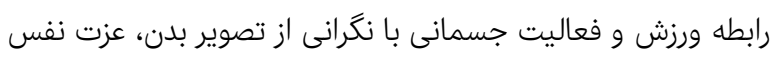

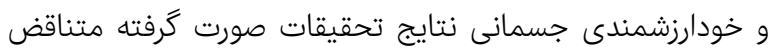

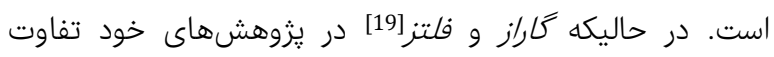
معنىدارى ميان گروه ورزشكار و غيرورزشكار در زمينه عزت مالت نفس

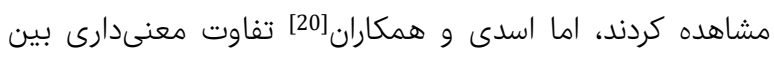
عزت نفس دانشجويان ورزشكار و غيرورزشكار گزارش نكردند.

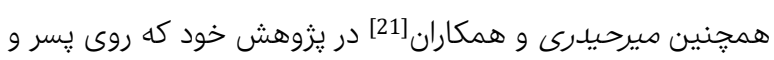

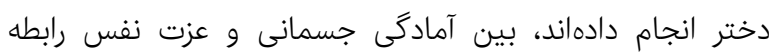

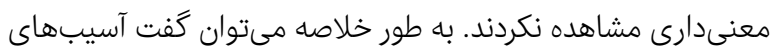


يافتهها

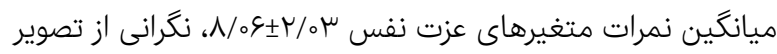

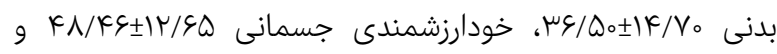

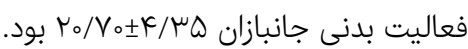

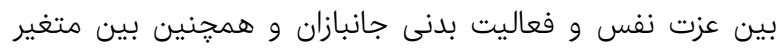

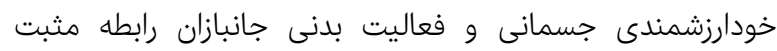

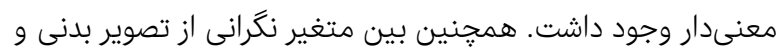

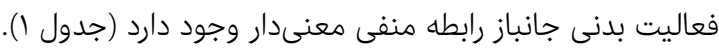

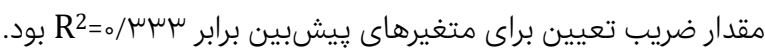

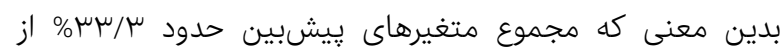

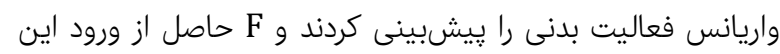

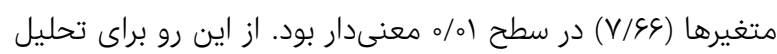

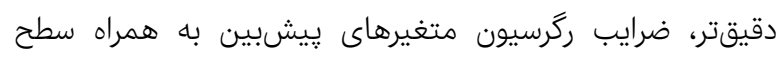

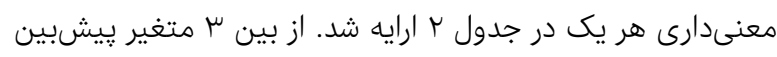

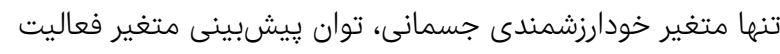

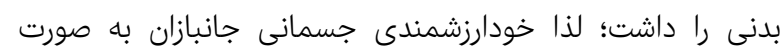
مثبت، فعاليت بدنى آنها را ييشبئ ردأينى كرد.

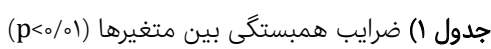

\begin{tabular}{|c|c|c|c|c|}
\hline$\varepsilon$ & $\mu$ & $r$ & 1 & متغير \\
\hline & & & 1 & ا. عزت نفس \\
\hline & & 1 & $-0 / 0.4$ & r. نكرانى از تصوير بدنى \\
\hline & 1 & $-\circ / \Delta S D$ & $\circ / \& \Delta \Lambda$ & ". خودارزشمندى جسمانى \\
\hline 1 & - $/ \Delta \mu r$ & $-\circ / \mu^{\mu} \gamma \mu$ & $0 / k F Y$ & ع. فعاليت بدنى \\
\hline
\end{tabular}

جدول r) نتايج تحليل ركرسيون קندمتغيره به منظور ييشبينى متغير فعاليت

\begin{tabular}{|c|c|c|c|c|}
\hline $\mathbf{p}$ & $t$ & $\boldsymbol{\beta}$ & B & متغيرهاى ييشبين \\
\hline$\% 9 \mu$ & $1 / V 1$ & O/TKG & -/QYY & عزت نفس \\
\hline ०/918 & $-0 / 10 \mathrm{~V}$ & $-\circ / / Y \circ$ & $-\circ / \circ \Delta$ & نكرانى از تصوير بدنى \\
\hline $0 \% 09$ & T/VYG &.$/ 410$ &.$/|k|$ & خودارزشمندى جسمانى \\
\hline
\end{tabular}

\section{بحث}

از عوارض هر جنكى، بروز مشكلات جسمى و روانى براى افرادى

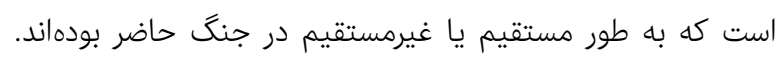

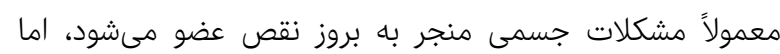

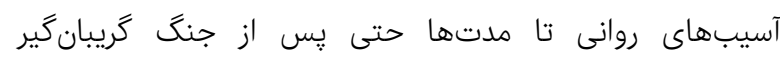

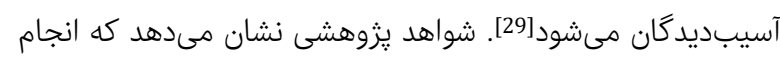

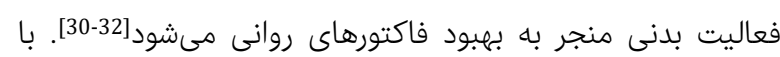

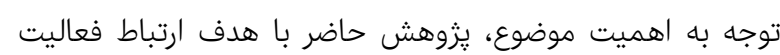

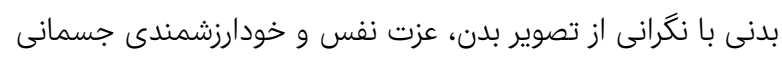

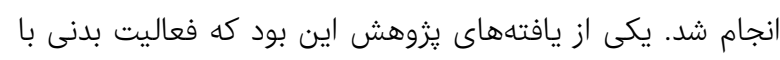

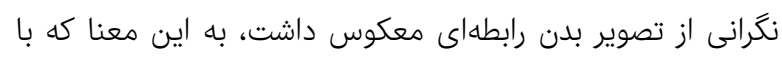

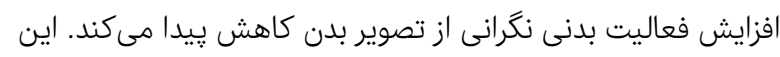

يرسشنامه عزت نفس: براى سنجش عزت وهن نفس، از مقياس 10

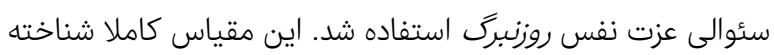

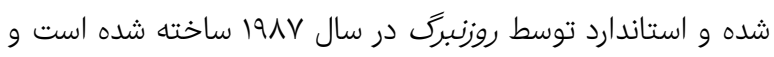

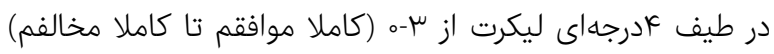

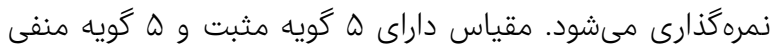

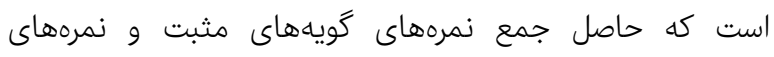
معكوسشده گويههاى منفى، ميزان عزت نفس فره فرد راه را مشخص

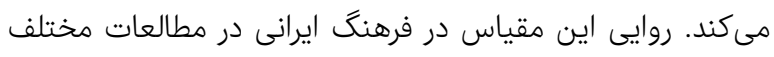

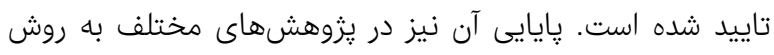

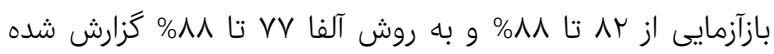

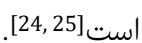

مقياس خودارزشمندى جسمانى Body Appreciation) Scale; BAS) همكاران ساخته شد و هدف آن اندازهيرى تصوير جسمانى مثبت دريت

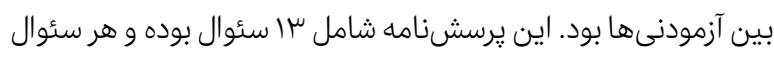

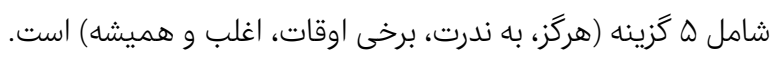

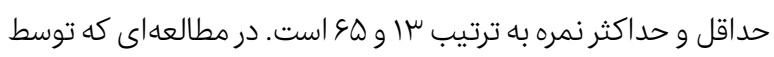

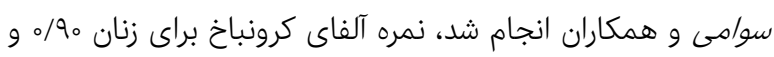

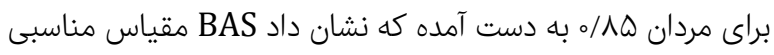

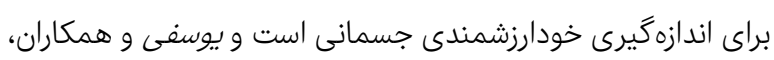
روايى و يايايى يرسشنامه را مطلوب گزارش كردهاند [26].

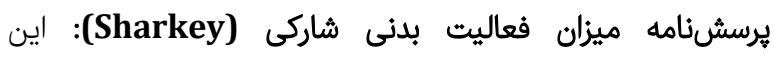
يرسشنامه براى ارزيابى ميزان فعاليت بدنى بوده و شامل ه سئوال هَزينهاى است كه براساس طيف ليكرت تهيه و تدوين شده است.

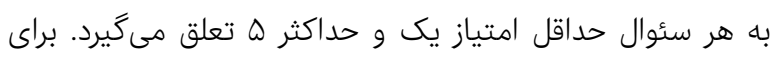

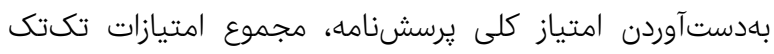

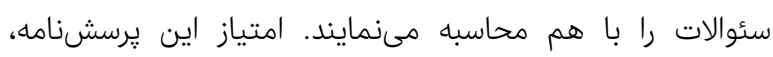

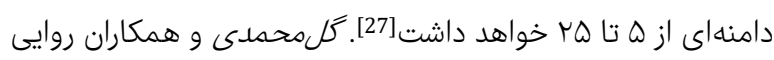

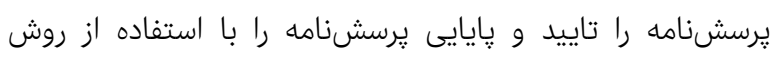

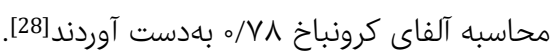

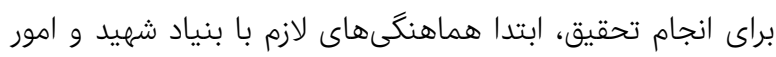

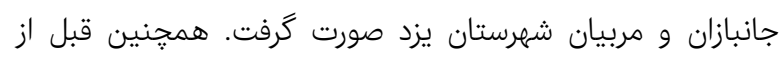

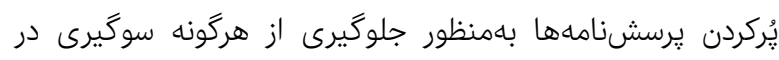

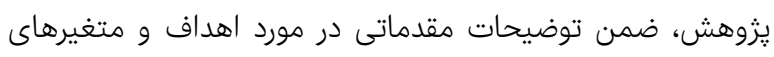

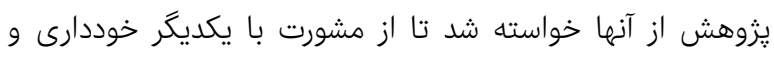

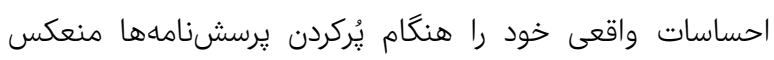

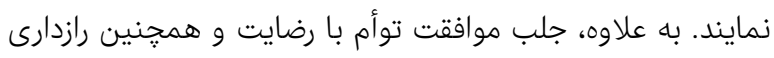

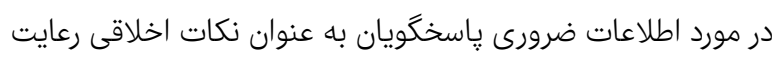

بهمنظور بررسى رابطه متغيرها، از ضريب همبستگى يِيرسون و براى

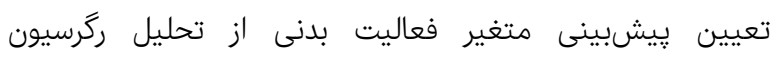

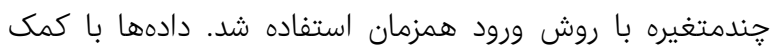
نرمافزار SPSS 23 تجزيه و تحليل شدند. 


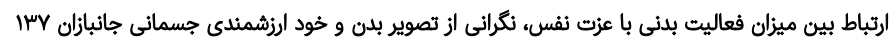

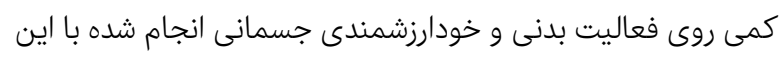

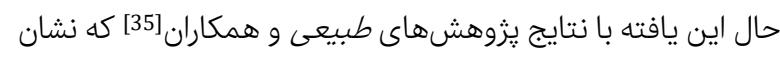

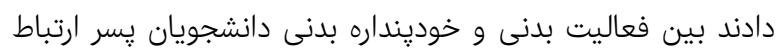
معنىدارى وجود دارد، همسو است. به علاوه يافتهها با نتايج وليج

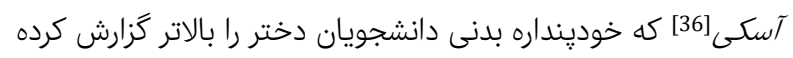

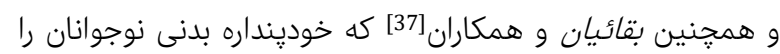

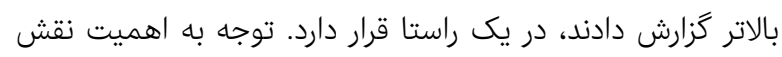

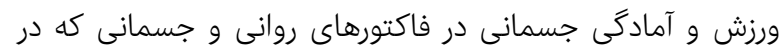

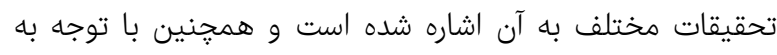

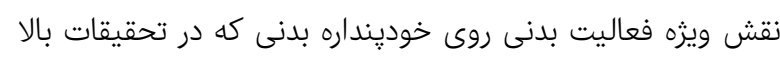

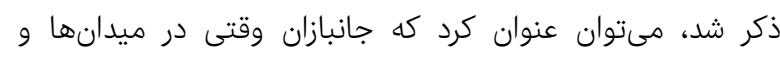

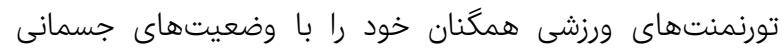

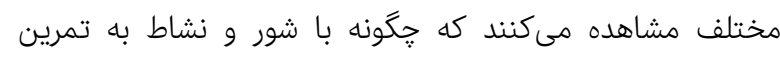

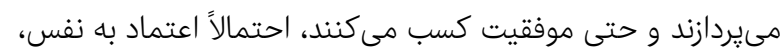

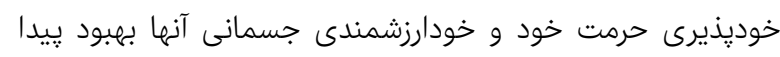

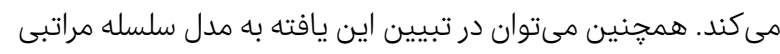

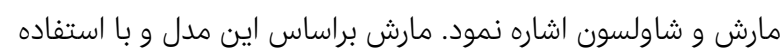

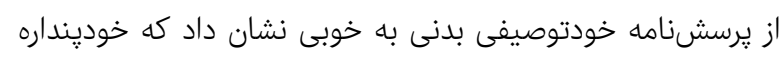

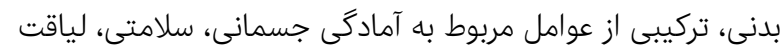

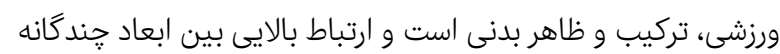

خودينداره بدنى و اجزاى آمادگى جسمانى وجود دارد [38].

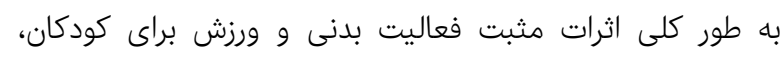

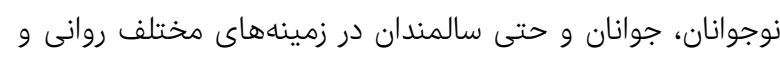

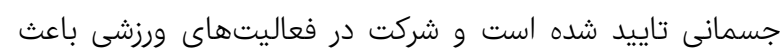

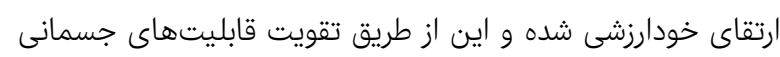

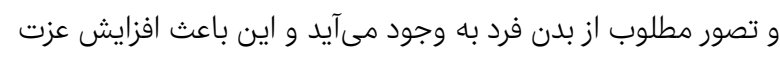

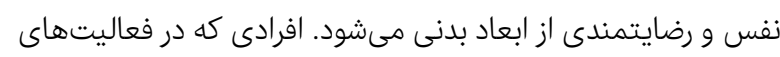

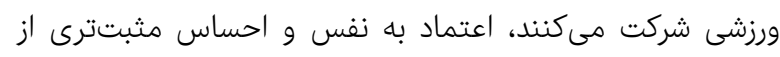
شايستخىهاى جسمانى دارند و در واقع يكى از كاركردهاى مهم

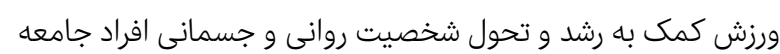
و تحكيم معاشرت جمعى و تسهيل دستيابى به رشد مسئوليت

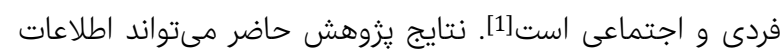

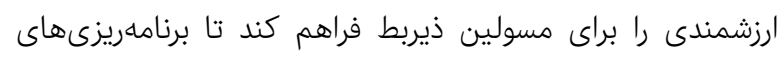

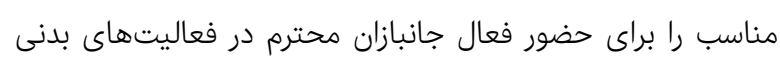

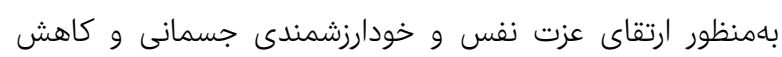
متغير نكرانى از تصوير بدنى فراهم نمايند.

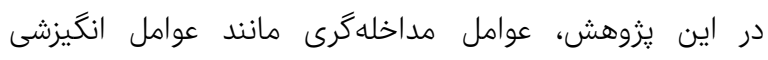

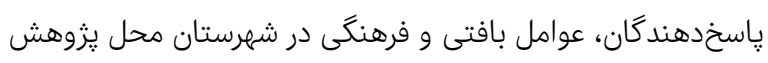

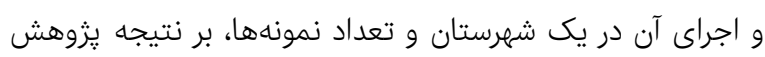
تاثيرگذار بوده است. نظر به إنه محدوديتهايى مانند محدوديت

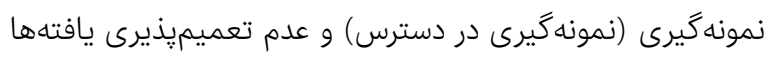

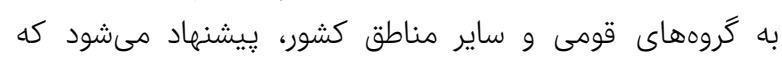
يزوهشى با محدوده جغرافيايى و جامعه آمارى ديكر انجام شود.

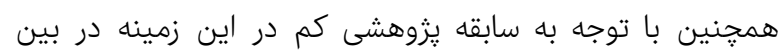

نتايج با يافتههاى حسينى سربازى و همكاران[33] كه نشان دادند

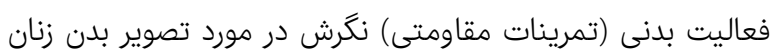

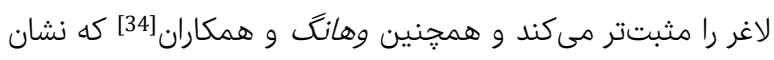

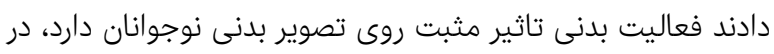

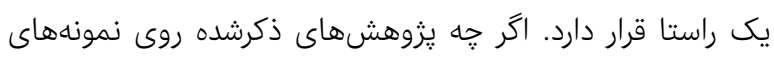

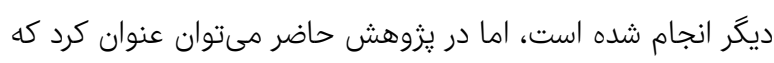

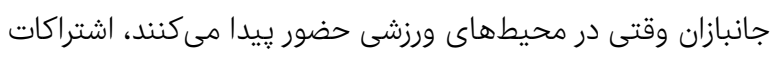

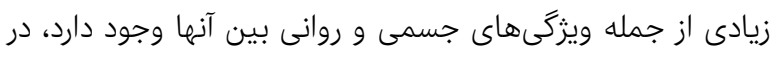

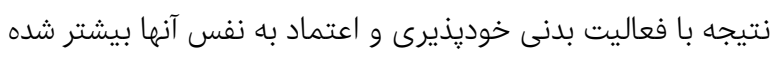

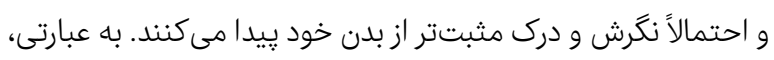

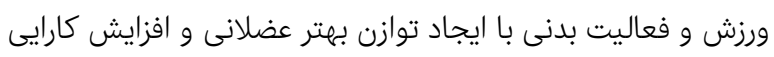

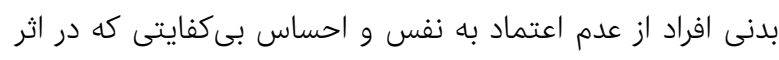

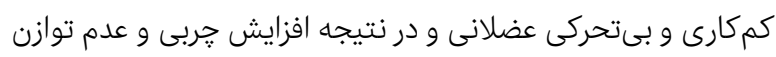

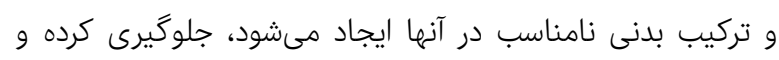

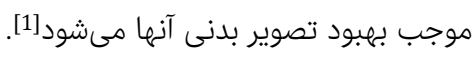

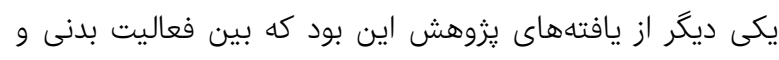

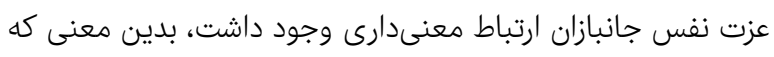

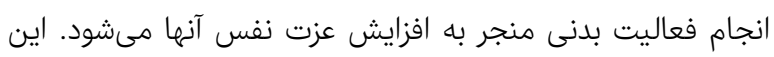

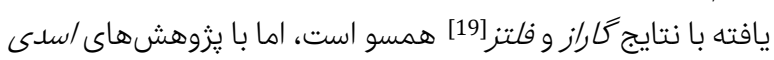

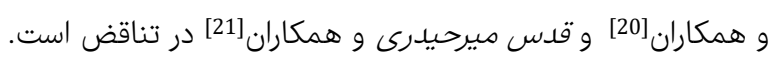

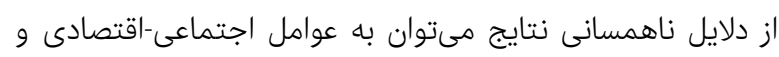

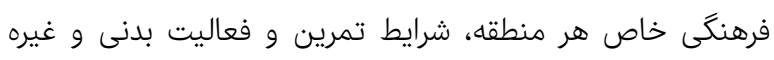

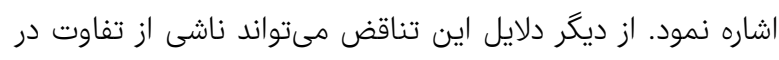

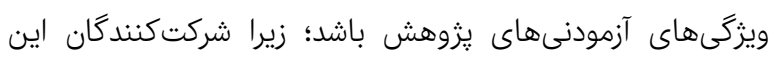

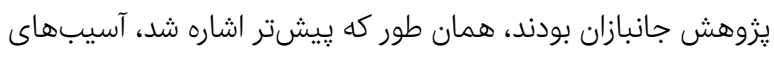

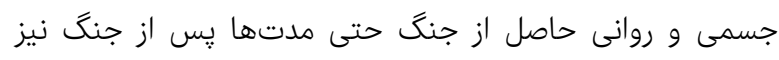

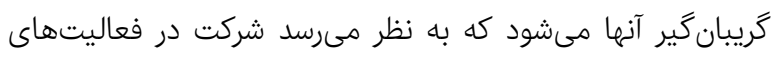

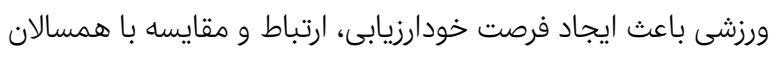

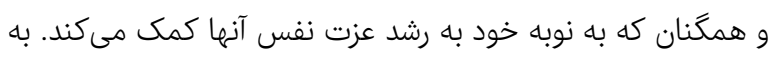

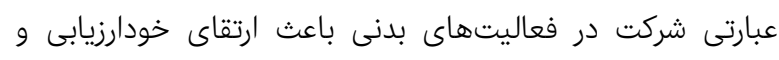

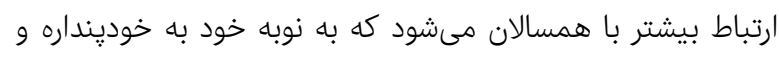
يذيرش خودواقعبينانه از خود كمك بيشترى نموده و باعث افزايش

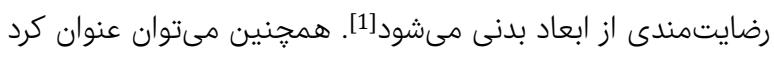

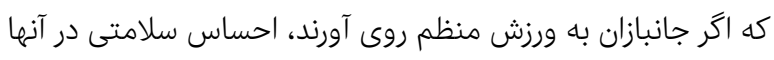

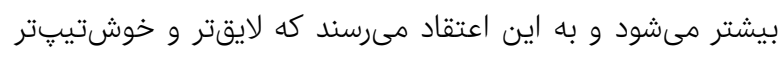

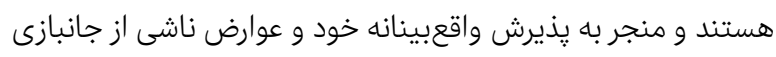

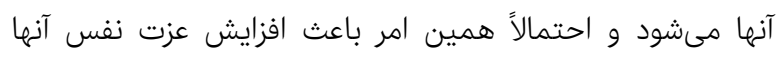

مى شود. همجنين نتايج يزوهش حاضر نشان داد كه بين فعاليت بدنى و

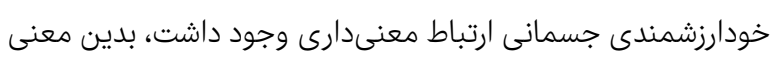
كه فعاليت بدنى تاثير مثبت بر خودارزشمندى جسمانى دارد. به إنه

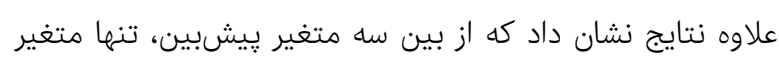

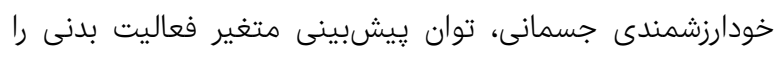

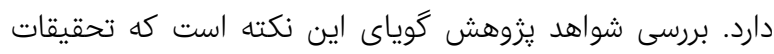


7- Gilbert N, Meyer C. Fear of negative evaluation and the development of eating psychopathology: A longitudinal study among nonclinical women. Int J Eat Disord. 2005;37(4):307-12.

8- Webster J, Tiggeman M. The relationship between women's body satisfaction and self-image across the life span: The role of cognitive control. J Genet Psychol. 2003;164(2):241-52.

9- Markham A, Thompson T, Blowing A. Determinants of body-image shame. Personal Individ Differ. 2005;38(7):1529-41.

10- Baghernezhad Hesari F, Morowatisharifabad MA, Sharifzadeh G, Miri MR, Vahdaninia V, Dastgerdi R. Predictive factors of self-esteem in adolescent girls. PAYESH. 2019;18(6):537-45. [Persian]

11- Ghaffari K, Hosseini SS, Davoodi H, Lak S. Investigate the relationship between self-esteem and organizational performance and job satisfaction among managers. J New Approach Educ Manag. 2018;9(1):208-23. [Persian] 12- Zaki MA. Standardization of self-worthiness questionnaires among male and female high school students in Isfahan. Psychol Method Model. 2012;2(7):6385. [Persian]

13- Arefi M, Mohammadi P. The relationship between selfworth and communication styles and marital adjustment in women. Shenakht J Psychol Psychiatry. 2016;2(4):1122. [Persian]

14- Zarepoor F, Kamali M, Alagheband M, Gheisari M, Sarlak SH. Evaluation of depression and its relationship to exercise in women over 20 years. J Shahid Sadoughi Univ Med Sci.2012;20(1):64-72. [Persian]

15- Khoroush M, Ehsani M, Tondnevis F. Relationship between physical activity and job burnout and achievement motivation in sports experts of the physical education organization of the Islamic Republic of Iran. J Motor Sci Sports. 2010;7(14):141-50. [Persian]

16- Borrer KT. Physical activity in the prevention and amelioration of osteoporosis in women. Sports Med. 2005;35:779-830.

17- Dunton G, Schneider M, Graham D, Cooper D. Physical activity, fitness, and physical self-concept in adolescent females. Pediatr Exerc Sci. 2006;18(2):240-51.

18- Marsh HW, Chanal JP, Sarrazin PG. Self-belief does make a difference: A reciprocal effects model of the causal ordering of physical self-concept and gymnastic performance. J Sports Sci. 2006;24(1):101-11.

19- Garza DL, Feltz D. Effect of selected mental practice on performance, self-efficacy, and competition confidence of figure skater. Sport Psychol. 1998;12:1-15.

20- Asady SK, Tasaddoghi Z, Tavakoli, M. Comparing selfesteem and self-concept of athletic and non-athletic students and finding a relationship between these two variables. Ann Appl Sport Sci. 2013;1(1):28-33.

21- Ghods Mirheydari SA, Takalli H, Abdollahi M, Gharayegh Zandi H, Mashhouri M, Ghods Mirheydari SB. The comparison and relationship between self-esteem and physical fitness in male and female students of Tehran university. Dev Motor Learn. 2010;(5):85-100. [Persian]

22- Littleton HL, Axsom D, Pury CLS. Development of the body image concern inventory. Behav Res Ther. 2005;43(2):229-41.

23- Bassak Nejad S, Ghafari M. The relationship between body dysmorphic concern psychological disorders and among university students. J Behav Sci. 2008;1(2):17987. [Persian]

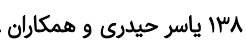
جانبازان كشور، يِيشنهاد مى شيود تا در مطالعات آتى، علاوه بر كنترل

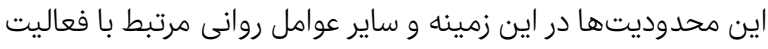

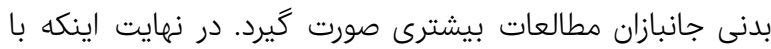

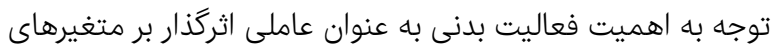

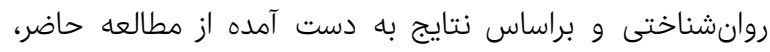

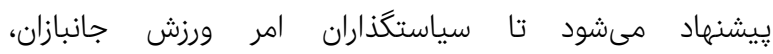
برنامهريزىهاى مناسب را براى حضور فعال جانبازان محترم در

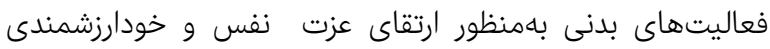
جسمانى و كاهش متغير نگرانى از تصوير بدنى فراهم نمايند.

\section{نتيجه گيرى}

بين فعاليت بدنى با عزت نفس، خودارزشمندى جسمانى و نكرانى

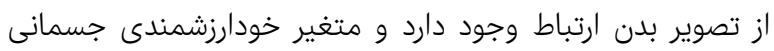
توان پيشبينى متغير فعاليت بدنى را دارد.

تشكر و قدردانى: از كليه جانبازان، مسئولين بنياد شهيد كه ما را در انجام

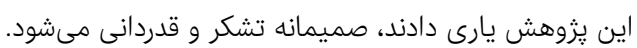

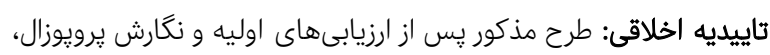

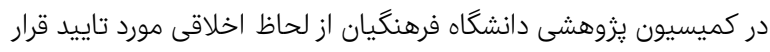
كرفته است. تعارض منافع: هيج گَونه تعارض منافع گَزارش نشد.

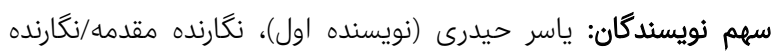

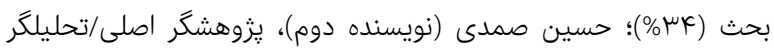

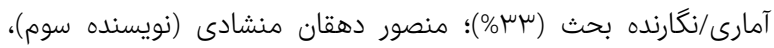

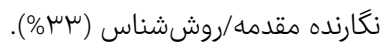

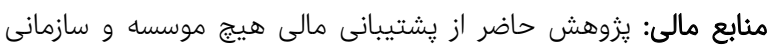

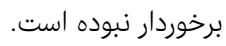

\section{منابع}

1- Aghdasi MT, Samadi H, Ghari B. Comparison of body dimensions satisfaction and sociophysical anxiety between veteran athletes of team and single sports. Iran J War Public Health. 2015;7(3):167-73. [Persian]

2- Samadi H, Mousavi SH, Shirvani H. The effect of internal attentional focus instructions and various distances of external attention on the static and dynamic balance of the chemical veterans with movement impairment. J Mil Med. 2019;21(6):596-605. [Persian]

3-Sheikh M, Moslemi Nejad M. Comparison of body image anxiety of athletic and nonathletic elder women: The mediator role of body index with relation to self-respect and body image anxiety. J Psychol Sci. 2016;15(57):98112. [Persian]

4- Murray KM, Byrne DG, Rieger E. Investigating adolescent stress and body image. J Adolesc. 2011;34(2):269-78.

5- Segal BN, Bradford A, Meston CM. The association between body esteem and sexual desire among college women. Arch Sex Behav. 2009;38(5):866-72.

6- Khanjani Z, Babapour J, Saba G. Investigating mental status and body image in cosmetic Surgery applicants in comparison with non-applicants. J Shahid Sadoughi Univ Med Sci. 2012;20(2):237-48. [Persian] 
ارتباط بين ميزان فعاليت بدنى با عزت نفس، نكرانى از تصوير بدن و خود ارزشمندى جسمانى جانبازان وسا

anxiety in obese women. Nurs Midwifery Paramed. 2019;4(3):16-24. [Persian]

32- Jalalvand S, Yousefi B, Farahani A. Study of relationship between physical activity and self-esteem, physical self-esteem and socio-physical anxiety in male and female high school students. Res Sports Life Sci. 2012;2(5):27-37. [Persian]

33- Hosseini Sarbazai M, Badami R, Taghian F. Effect of strength exercise on physical appearance, eating disorder and sexual function in slender females. Sport Psychol Stud. 2017;6(20):137-48. [Persian]

34- Huang JS, Norman GJ, Zabinski MF, Calfas K, Patrick K. Body image and self-esteem among adolescents undergoing an intervention targeting dietary and physical activity behaviors. J Adolesc Health. 2007;40(3):245-51. 35- Tabiee S, Vagharseyyedin SA, Salmani-Mud M, Hosseini M. Relationship of physical self-concept and athletic performance in male medical students in Birjand university of medical sciences. J Birjand Univ Med Sci. 2018;25(1):52-61. [Persian]

36- Asci FH. The effects of physical fitness training on trait anxiety and physical self-concept of female university students. Psychol Sport Exerc. 2003;4(3):255-64.

37- Baqaian M, Bahram A, Khalaji $\mathrm{H}$. The effect of gender and level of physical activity on the physical self-concept of middle school students. Educ Innov. 2013;12(47):8294. [Persian]

38- Marsh HW. Construct validity of physical selfdescription questionnaire responses: Relations to external criteria.J SportExerc Psychol.1996;18(2):111-31.
24- Rosenberg M, Schooler C, Schoenbach C, Rosenberg F. Global self-esteem and specific self-esteem: Different concept, different outcomes. Am Sociol Rev. 1995;60(1):141-56.

25- Masoudnia A. The Comparison of coping styles of stress of students with different self-esteem. J Educ Sci Psychol. 2008;15(1):81-98. [Persian]

26- Yousefi B, Hassani Z, Shokri O. Reliability and factor validity of the 7-item social physique anxiety scale (SPAS7) among university students in Iran. World J Sport Sci. 2009;2(3):201-4.

27- Khodabakhshi Koolayee A, Sabzi S, Shahdadi H, Mohamadi F. The Comparison of brain cognition function between active and inactive elderlies male in nursing home (a case-control study in Tehran). Commonity Health. 2017;4(4):302-9. [Persian]

28- Golmohammadi B, Kashani V, Mokaberian M. Persian psychometric properties of homework self-efficacy scale for everyday elderly activities. J Clin Psychol. 2015;7(2):87-100. [Persian]

29- Nabatian E, Ghamarani A, Zakerian M, Mahdizadeh I. Relationship between spiritual health with quality of life veterans and disabled Birjand. Iran J War Public Health. 2013;5(2):35-9. [Persian]

30- Gammage KL, Hall CR, \& Ginis KAM. Self-Presentation in Exercise Contexts: Differences between high and low frequency exercisers. J Appl Soc Psychol. 2004;34(8):1638-51.

31- Babaei Bonab S, Nobaran E, Derakhti R. Study of the relationship between regular physical activity and social 